\title{
Electromagnetic Simulations of Mechanical Imperfections for Accelerator Cavities
}

\author{
Anıl Karatay and Fatih Yaman ${ }^{(1)}$
}

\begin{abstract}
Effects of surface roughness and transversal cell misalignments on the performance of elliptical accelerator cavities are studied in this article. A high- $\beta$, 9-cell elliptical cavity, whose $\pi$-mode resonates at $3.9 \mathrm{GHz}$, is designed to investigate imperfections. The considered frequency is chosen to observe variations of fundamental accelerating cavity parameters, wake potentials, and wake impedances more clearly by using relatively small structures. Moreover, 3-cell elliptical cavities having $\pi$-mode at 2 and $3.9 \mathrm{GHz}$ are designed to confirm the 9-cell cavity results. The undesired effects caused by the considered mechanical imperfections are simulated for an ultra-relativistic bunch in the parameter scope of a realistic scenario. In particular, Huray's snowball model, which is a scattering-based surface roughness approach developed for microstrip lines, is employed to determine the effects of the surface roughness on the accelerator cavities. Surface roughness due to the fabrication process is expressed as a surface impedance, and the required equivalence between the surface roughness and surface impedance concept is achieved. Significant computational efficiency is observed by using the surface impedance concept with Huray's snowball model in the simulations. Experimental verification of certain parameters is included for an elliptical cavity having high cell-to-cell coupling at $3.9 \mathrm{GHz}$.
\end{abstract}

Index Terms-Elliptical cavity, misalignment, particle accelerator, roughness, wakefield.

\section{INTRODUCTION}

$\mathbf{F}$ ABRICATION of cavities within the desired mechanical tolerance limits is one of the most challenging problems for efficient power transfer from high-frequency sources to the beams in modern particle accelerators. The main difficulties for this task appear in the accurate assembling of the half-cells and the etching process. Therefore, detection of misalignments and excessive surface roughness, as well as quantification of the related destructive effects, are critical issues, especially for long multi-cell elliptical cavities. In this context, we will focus on a commonly seen misalignment at the center of the cells by considering a prototype elliptical cavity in the first part of the article. Negative effects of such a scenario on the accelerator cavity design parameters, for example, $R_{\mathrm{sh}} / Q$, $E_{\text {peak }} / E_{\text {acc }}, B_{\text {peak }} / E_{\text {acc }}$, and $G$ (geometry factor) will be illustrated. Furthermore, the impact of the defect location onto the

Manuscript received June 5, 2019; revised August 8, 2019 and September 22, 2019; accepted September 26, 2019. Date of publication October 2, 2019; date of current version November 18, 2019. This work was supported by the İzmir Institute of Technology Scientific Research Program (BAP) under Grant IYTE0299.

The authors are with the Department of Electrical and Electronics Engineering, İzmir Institute of Technology, 35430 İzmir, Turkey (e-mail: fatihyaman@iyte.edu.tr).

Color versions of one or more of the figures in this article are available online at http://ieeexplore.iee.org.

Digital Object Identifier 10.1109/TNS.2019.2945138

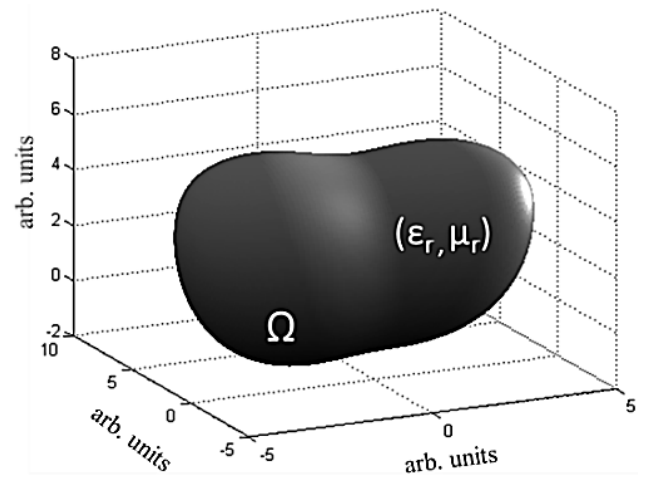

Fig. 1. Resonant cavity structure.

electric and magnetic fields will be analyzed for the cavity parameters. In the second part of the article, the applicability of the surface impedance approach and Huray's snowball model [1]-[4] for cavities are shown on a rectangular resonator structure. Afterward, wake potentials and coupling impedances [5]-[7] are computed via the Computer Simulation Technology (CST)-wakefield solver [8]. At this point, it is worth mentioning that one needs to use highly dense grids for the computational domains and a powerful computer in order to observe electromagnetic effects of surface imperfections via a 3-D full Maxwell solver software. However, we will tackle this problem with the help of an impedance concept such that it can be used as a tool to model the surface roughness on accelerator cavity walls [9].

In the literature, imperfections of cell length, radius, and shape deformations of the elliptical cavity for 1.3 and $1.82 \mathrm{GHz}$ were investigated in [10] and [11], and tolerance limits of the elliptical cavity for the $\pi$-mode frequencies were determined. In our study, the investigated frequency of the $\pi$-mode is increased to $3.9 \mathrm{GHz}$ in order to observe the effects in a more clear form. The imperfection results agree with those obtained by two 3-cell cavities whose $\pi$-modes resonate at 2 and $3.9 \mathrm{GHz}$, which indicate that the observations are independent of the operating frequency, the number of cells, and the size of the cavity for a similar parameter range. These simulations are based on finding the eigenvalue and eigenvector solutions of arbitrarily shaped structures like in Fig. 1.

The following continuous eigenvalue problem:

$$
\begin{aligned}
\left(1 / \mu_{r}\right) \nabla x \nabla x \mathbf{E}(x, y, z) & =\tau^{2} \epsilon_{r} \mathbf{E}(x, y, z), \quad x, y, z \in \Omega \\
\tau^{2} & =\omega^{2} / c^{2}
\end{aligned}
$$

in terms of electric field $\mathbf{E}$, can be obtained via the substitution of Maxwell-Ampere equation to the resulting curl of 
Maxwell-Faraday representation for the source-free bounded domain $\Omega$ enclosed by the surface $\partial \Omega$. Here, $\tau$ is the resonant frequency related parameter to be found, $c$ stands for the speed of light, $\epsilon_{r}$, and $\mu_{r}$ are relative permittivity and permeability of the medium inside the cavity, respectively. The bold characters represent the vector quantities. Furthermore, the following equation, which originates from Gauss's law for source-free closed regions:

$$
\nabla \cdot \mathbf{E}=0
$$

has to be satisfied in the domain $\Omega$. The boundary condition for this problem guarantees that the tangential component of the electric field vanishes on the surface $\partial \Omega$ due to physical constraints, that is

$$
\mathbf{n} \times \mathbf{E}=0
$$

where $\mathbf{n}$ is the normal vector. The stated eigenvalue problem is evaluated via the finite-element method (FEM) with the aid of a well-known program CST-eigenmode solver [8]. Using tetrahedral meshes for a prototype elliptic cavity, we obtain the resonant frequencies, corresponding field distributions, and results of alignment errors from an electromagnetic point of view.

The organization of the article is as follows. Section II is devoted to express the design of a prototype high- $\beta$ elliptical cavity for ultra-relativistic beams. Afterward, we present numerical results related to cell misalignments for the proposed structure. In Section III, the surface impedance concept is explained, and the efficiency of the method for a test cavity is presented. Then, wake potential and impedance calculations using surface impedance approach are illustrated. Next, the experimental results on the frequency and geometry factor of a 3-cell 3.9-GHz elliptical cavity with high cell-to-cell coupling are given. The summary and the concluding remarks are given in Section VII. One should note that the superconductivity breakdown is neglected throughout this article.

\section{CAVITy Design}

Before modeling the imperfections, an ideally fabricated prototype elliptical cavity is prepared via an eigenmode solver. The resonant frequencies and the electromagnetic fields are calculated by omitting an excitation source inside the cavity according to the problem statement defined in (1)-(3). The geometry of the 9-cell cavity, whose length $\sim 34.5 \mathrm{~cm}$ (without beam pipes) with $\sim 6.78-\mathrm{cm}$ transverse extends, is given in Fig. 2 on the top of the electric-field plot.

As can be seen from the variation of the electric-field magnitude on the beam axis, the proposed cavity satisfies the desired field flatness, and it can be used to accelerate particles in the $\pi$-mode since the maximum values of the fields are well-located in the middle of each cell from \#1 to \#9. Furthermore, fundamental parameters of the prototype cavity, listed in Table I, are in the scope of typical accelerating elliptical cavity parameters used in practice.

Here, we note that $R_{\mathrm{sh}} / Q$ corresponds to the relative concentration of energy on the beam axis, $E_{\text {peak }} / E_{\text {acc }}$ is the ratio between the peak electric field and accelerating gradient, and

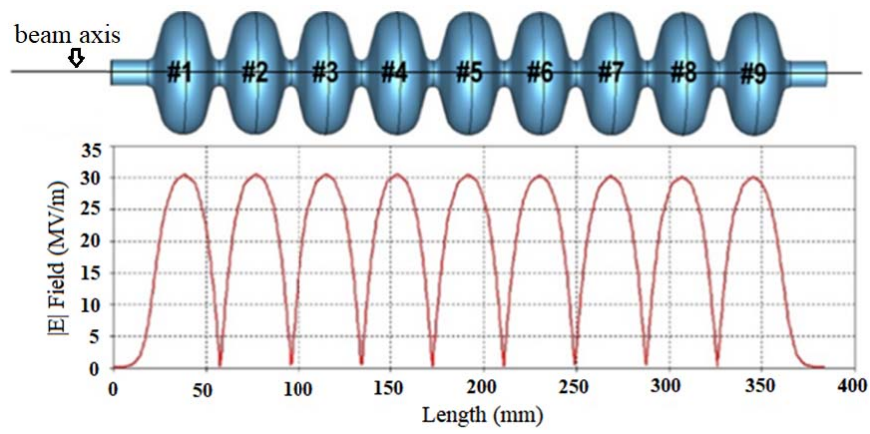

Fig. 2. Magnitude of the electric field at 3.9-GHz along the beam axis.

TABLE I

Fundamental CAVity Parameters

\begin{tabular}{cll}
\hline \hline Parameter & Value & Unit \\
\hline$\pi$-mode frequency & 3.9008 & $\mathrm{GHz}$ \\
$\mathrm{R}_{\text {sh }} / \mathrm{Q}$ & 1331.54 & $\Omega$ \\
$\mathrm{E}_{\text {peak }} /$ acc $_{\text {acc }}$ & 1.8393 & - \\
$\mathrm{B}_{\text {peak }} /$ accc $_{\text {acc }}$ & 4.284 & $\mathrm{mT} /(\mathrm{MV} / \mathrm{m})$ \\
$\mathrm{G}$ & 99.7124 & $\Omega$ \\
$\mathrm{k}_{\mathrm{cc}}$ & $0.2 \%$ & - \\
\hline \hline
\end{tabular}

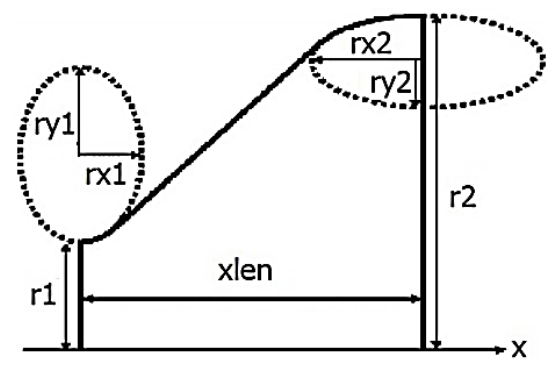

Fig. 3. Structure of an elliptical cavity.

$B_{\text {peak }} / E_{\text {acc }}$ is the ratio between the peak magnetic field and accelerating gradient. The geometry factor $G$ is the quality factor times surface resistance depending on the cavity mode and shape but not on its size [7].

The mentioned design considerations can be optimized by adjusting the shape parameters of the elliptical cavity, as indicated in Fig. 3. In this figure, we represent the parameters whose values are given in Table II for the inner half-cell geometry.

Note that two end half-cells of the cavity are chosen to be $0.1-\mathrm{mm}$ longer than the inner half-cells in the vertical axis in order to optimize the electric-field distribution inside the cavity.

Higher acceleration efficiency $\left(R_{\mathrm{sh}} / Q\right)$ is obtained for a smaller iris radius $(r 1)$ at the expense of a decrease in cellto-cell coupling. Therefore, the sensitivity of the field profile increases for geometrical changes in the cavity. Accordingly, the $r 1$ parameter is chosen smaller in this article compared to existing designs illustrated in [12] and [13] to lower the cell-to-cell coupling and to clarify the effect of misalignment on the cavity parameters. $B_{\text {peak }} / E_{\text {acc }}$ and $E_{\text {peak }} / E_{\text {acc }}$ of the newly designed cavity are very close to the parameters of the 
TABLE II

Dimensions OF THE ElLiptical CAVITY

\begin{tabular}{cl}
\hline \hline Parameter & Value $(\mathrm{mm})$ \\
\hline $\mathrm{r} 1$ & 7.25 \\
$\mathrm{r} 2$ & 33.91 \\
$\mathrm{rx} 1$ & 4 \\
$\mathrm{rx} 2$ & 12 \\
ry1 & 5 \\
ry2 & 12 \\
xlen & 19.167 \\
\hline \hline
\end{tabular}

TABLE III

Convergence of the Simulations

\begin{tabular}{cccc}
\hline \hline TeT Number & $\mathrm{H}_{\text {peak }}(\mathrm{mA} / \mathrm{m})$ & $\mathrm{Q}$ & $\mathrm{f}_{\pi}(\mathrm{GHz})$ \\
\hline 160.000 & 56517 & 15317 & 3.90069 \\
370.000 & 56412 & 15412 & 3.90072 \\
620.000 & 56288 & 15346 & 3.90074 \\
1.110 .000 & 56239 & 15339 & 3.90075 \\
1.820 .000 & 56224 & 15337 & 3.90075 \\
2.780 .000 & 56211 & 15336 & 3.90075 \\
4.640 .000 & 56210 & 15336 & 3.90075 \\
6.750 .000 & 56210 & 15336 & 3.90075 \\
\hline \hline
\end{tabular}

9-cell elliptical cavity in [14] while the operating frequency of that cavity is $1 \mathrm{GHz}$. On the other hand, the iris radius is much greater than the scaled version of our cavity. In this case, we can achieve much greater acceleration efficiency while the cavity designed in [14] has better cell-to-cell coupling $\left(k_{\mathrm{cc}}\right)$.

To see the dependence of the numerical results with respect to the chosen number of finite elements, the number of iterations have been carried out for different numbers of tetrahedral (TET) meshes until the peak value of the magnetic field, the quality factor of the cavity, and the resonant frequency of the $\pi$-mode become stable with respect to number of mesh cells. In other words, the numerical solution of the considered problem converges to the certain values for each parameter after approximately 4.5 million finite elements. The related behaviors of our simulations are illustrated in Table III, where the optimum number of TET meshes is used.

\section{Cell Misalignment Simulations}

In practice, elliptical cavities are fabricated via accurate assembling of the preconstructed half-cells using certain methods, for example, electron beam welding as in [14] and [15]. Therefore, the correct alignment of the half-cells is of critical importance for obtaining the desired geometry of elliptical cavities. Conversely, the misalignment is defined as the vertical deviation of the half-cells from their correct position. For instance, the centers of the two half-cells are not on the same axis, as can be seen in Fig. 4.

Figs. 5-8, depict variations of the fundamental accelerator cavity design parameters with respect to deliberately misaligned individual cells in the whole structure, for example, if the first cell is misaligned, then the remaining cells are correctly aligned. With this approach, we could determine

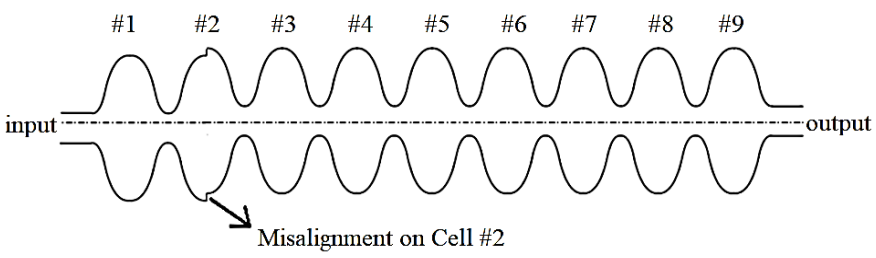

Fig. 4. Cell misalignment.

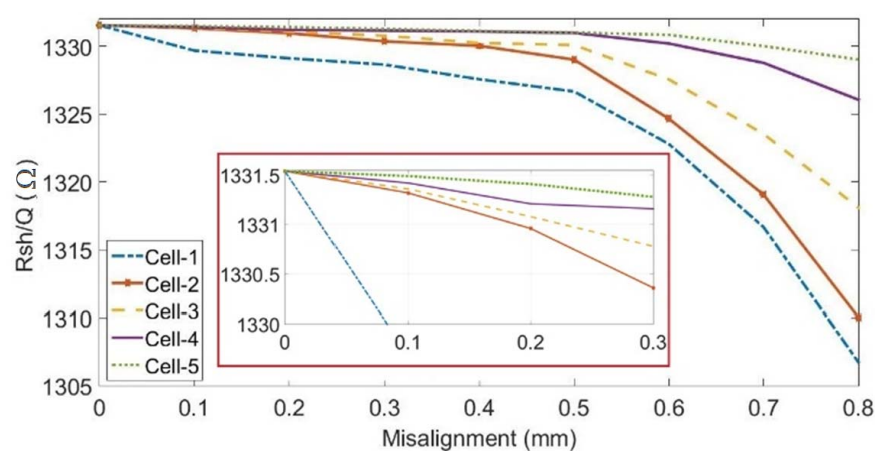

Fig. 5. Effect of cell misalignment on $R_{\mathrm{Sh}}$ over $Q$.

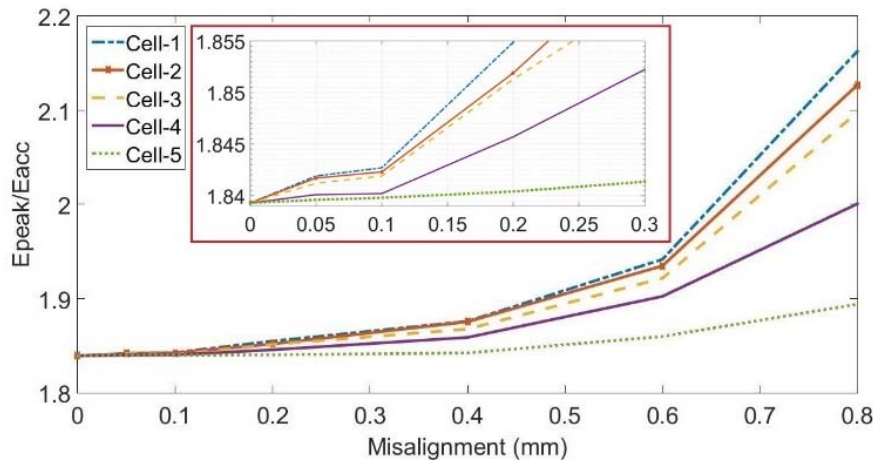

Fig. 6. Effect of cell misalignment on $E_{\text {peak }}$ over $E_{\text {acc. }}$

which cell has the strongest contribution to the instability and distinguish the effect of a certain cell to the considered parameter, separately. The misalignment deviations are gradually increased up to $0.8 \mathrm{~mm}$, which can be entitled as an exaggerated version of misalignment where the most common values up to $0.3 \mathrm{~mm}$ are specifically indicated with zoomed plots on the top of the main figures. Furthermore, the investigations presented for cells \#1 to \#4 are also valid for cells \#6 to \#9, since there is no excitation source in the computational domain for the studied homogeneous eigenvalue problem and the elliptical cavity has a circular symmetry transverse to the beam axis for the ideal geometry (without misalignment/roughness). In this case, simulating the first five cells of the structure is equivalent to simulating the entire structure.

We note that $E_{\text {acc }}$ is calculated by integrating on the beam axis. However, in the misaligned structure, the beam axis is not centered in all cells, as in Fig. 4. Our assumption is that the integration line is precisely centered in the output region and not exactly centered in the input region. Since the integration line also indicates the axis at which the beam will move, it must not contain a discontinuity. 


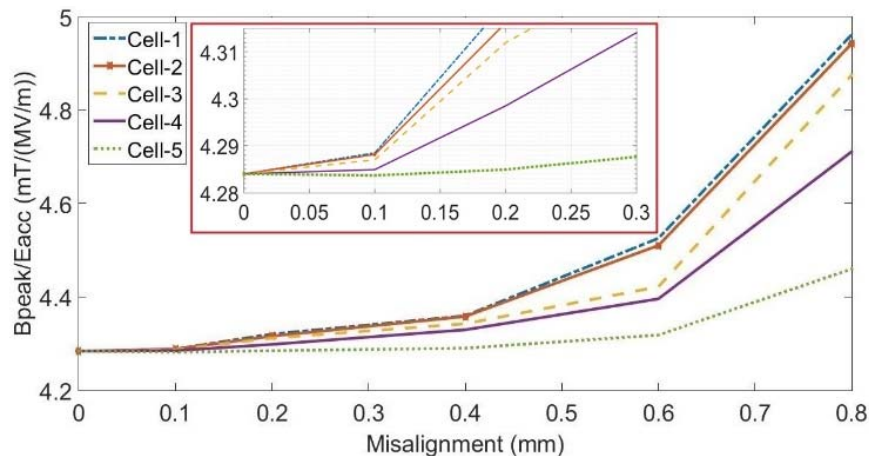

Fig. 7. Effect of cell misalignment on $B_{\text {peak }}$ over $E_{\text {acc. }}$.

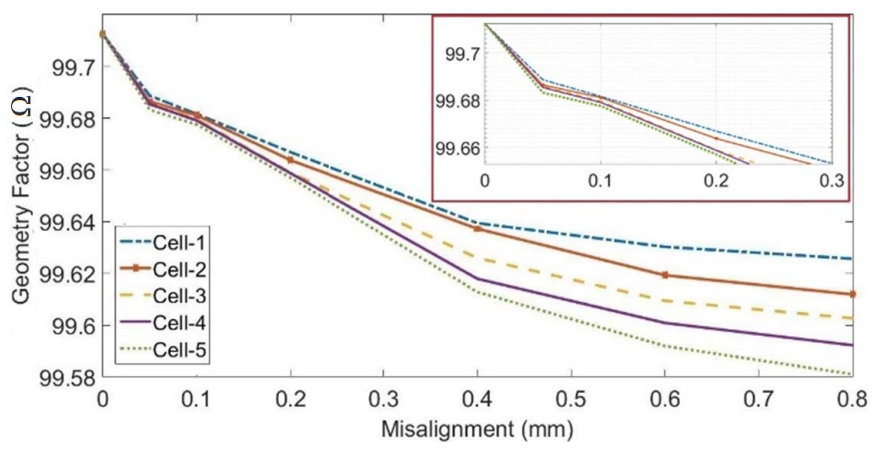

Fig. 8. Effect of cell misalignment on the geometry factor.

In Figs. 5-7, it is clearly observed that misalignment in the end cells (\#1 or \#9) has the most dominant effect on $R_{\mathrm{sh}} / Q, E_{\text {peak }} / E_{\text {acc }}$, and $B_{\text {peak }} / E_{\text {acc }}$ compared to the remaining cells. Additionally, there exists a certain pattern such that the numbers of misaligned cells and the magnitude of their effects on the parameters are inversely related, for example, \#5 has the weakest effect. On the other hand, this behavior is reversed in Fig. 8, such that the middle cell (\#5) has the strongest effect on the geometry factor. To increase the efficiency of the acceleration, the values of $R_{\mathrm{sh}} / Q$ and $G$ should be high, whereas $E_{\text {peak }} / E_{\text {acc }}$ and $B_{\text {peak }} / E_{\text {acc }}$ are as low as possible. In this frame, the fundamental cavity parameters deteriorate from their optimal values when the magnitude of the misalignment increases according to the figures.

Now, let us assume there is a misalignment on the mid-cell (\#5) of the cavity. Both electric (top) and magnetic (bottom) fields deteriorate in the fifth cell, as can be seen in Fig. 9. Principally, magnitudes of the electric field along the beam axis and magnetic field on the cavity walls are decreasing in the mid-cell while the fields tend to increase in perfectly aligned cells even though the integral of the electric field in the acceleration direction remains the same or slightly decreases. Therefore, we can conclude that this mechanism leads to a rise of $E_{\text {peak }} / E_{\text {acc }}$ and $B_{\text {peak }} / E_{\text {acc }}$ in Figs. 6 and 7.

Fig. 10 shows the increment of the peak electric field along the beam axis for a misaligned cell in a clearer way. Here, the red curve indicates the result of cell misalignment at cell-1, the green curve shows the misalignment at cell-3, and the blue curve corresponds to cell-5.

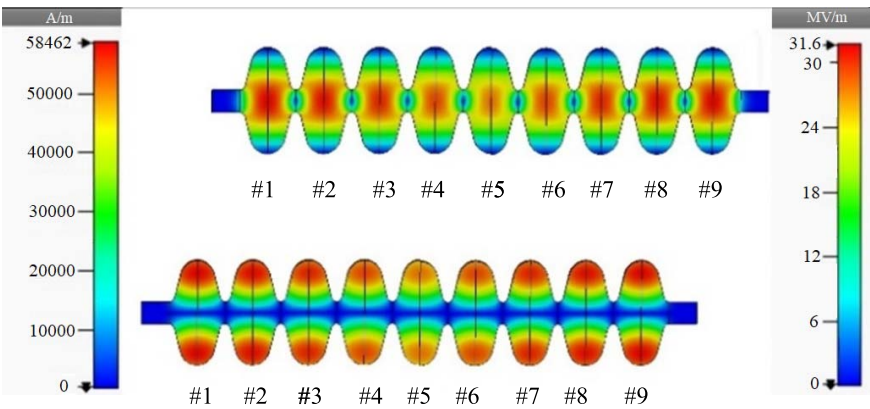

Fig. 9. Representation of electric and magnetic field with misalignment on cell-5.

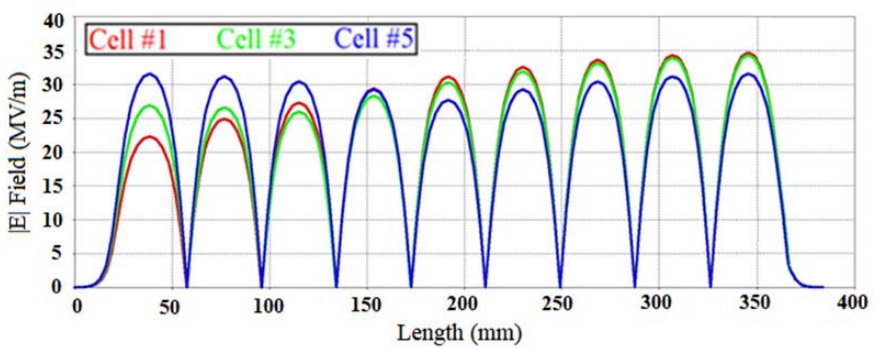

Fig. 10. Effect of cell misalignment on the electric field on the beam axis.

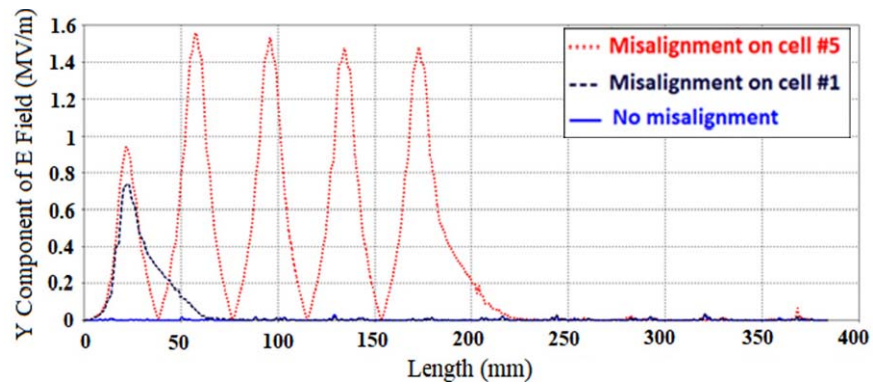

Fig. 11. Transverse electric field around the iris region.

As can be seen in Fig. 4, the beam axis does not pass through the exact centers of all cells due to the misalignment. Accordingly, the trajectory of the particles shifts close to the iris walls of the misaligned cells, and transverse kick occurs since the normal component of the electric field near the iris walls starts to play a more dominant role. Suppose that there is a misaligned cell in the transverse $y$-axis, while the $\mathrm{x}$-axis corresponds to the longitudinal direction of particle propagation. The particles approach the iris region of the first cell only when there is an alignment error on the first cell. On the other hand, if the alignment error occurs on the fifth cell, the particle trajectory would be close to the iris region for the first five cells. Similarly, if there is a misalignment for the ninth cell, the particles approach the iris region in all of the cells, but in this case, the problem can be overcome by centering the beam axis to the input rather than the output. In Fig. 11 one can observe the effect of the cell alignment error on the transverse electric field seen by the particle. The peaks observed in Fig. 11 occur around the iris regions of the 9-cell elliptical cavity. 


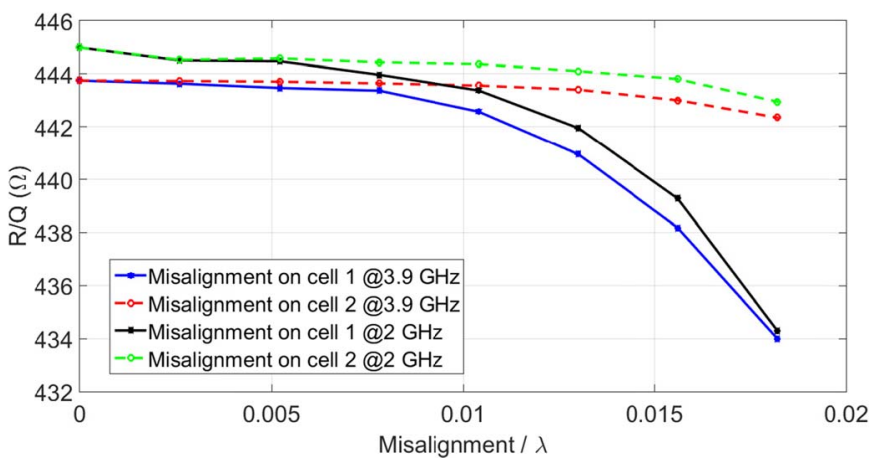

Fig. 12. Effect of cell misalignment on $R_{\mathrm{sh}} / Q$ for the 3-cell elliptical cavities.

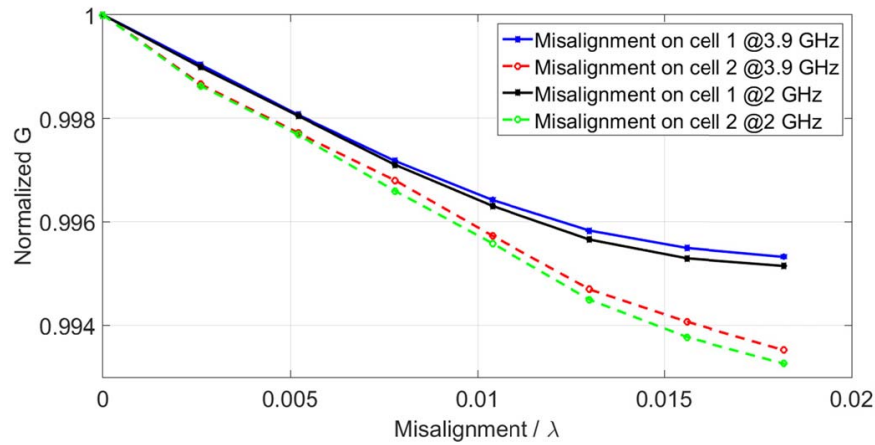

Fig. 13. Effect of cell misalignment on $G$ for the 3-cell elliptical cavities.

As a next step, the 9-cell elliptical cavity, whose $\pi$-mode resonates at $3.9 \mathrm{GHz}$ is scaled to the $2 \mathrm{GHz}$ ( $\pi$-mode) 3 -cell cavity in order to observe the behaviors of the fundamental cavity parameters with respect to cell misalignment for a lower frequency and a cell number. In addition, a 3-cell cavity at $3.9 \mathrm{GHz}$ is designed and these two 3-cell cavity results are compared such that the alignment errors are normalized to the wavelength $\lambda$. In this case, $R_{\mathrm{sh}} / Q$ and geometry factor parameters are reinvestigated for the 3-cell elliptical cavities.

It is clearly seen from Fig. 12. that $R_{\mathrm{sh}} / Q(\beta=1)$ decreases with the increase of cell misalignment for both $3.9-\mathrm{GHz} 3$-cell and 2-GHz 3-cell cavities. Similar to the 9-cell elliptical cavity result, the end cell is more important than the mid-cell on parameter $R_{\mathrm{sh}} / Q$. Similar behavior can be seen at frequencies 3.9 and $2 \mathrm{GHz}$. The geometry factor variation of the half-cell shift is illustrated in Fig. 13. Since the geometry factor values of the 3.9- and 2-GHz cavities are different, we normalize their values with their maximums (i.e., Normalized $G=G / G_{\max }$ ). As expected, the G-parameter deteriorates for the larger misalignments where the behavior of the geometry factor is consistent with the 9-cell case. Furthermore, the mid-cell has a more significant effect than end-cells on the geometry factor for both frequencies.

We also check the effect of misalignment on the frequency shift of the $\pi$-mode with 3-cell elliptical cavities. In Fig. 14, the resonant frequencies of the elliptical cavities shift slightly in the negative direction. However, the shift is sufficiently small such that it can be compensated via a tuner. For this reason, the frequency shift due to the cell misalignments is not the main concern. We observe that the effect of the misaligned

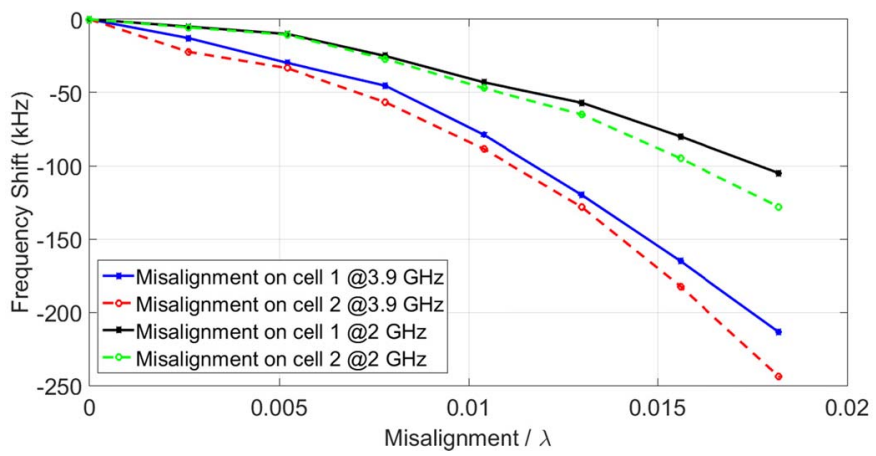

Fig. 14. Effect of cell misalignment on the resonant frequency.

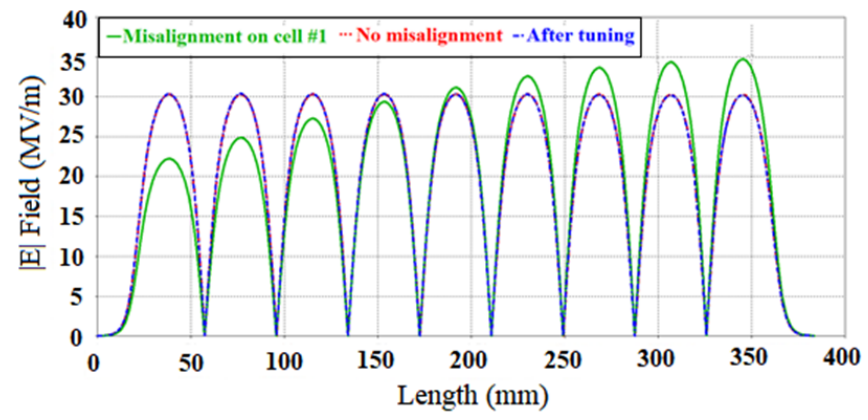

Fig. 15. Treatment of cell misalignment via perturbation tuning.

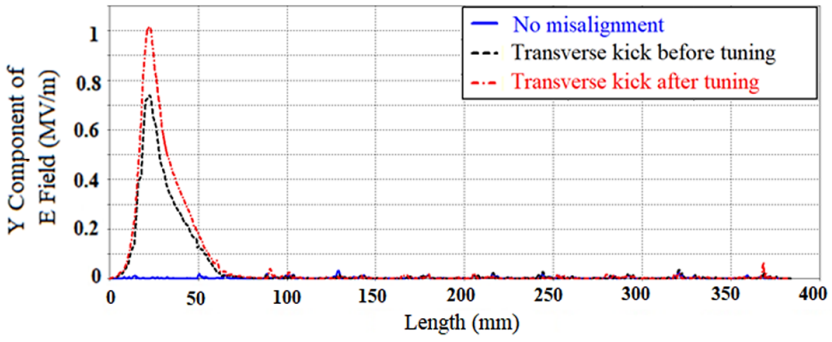

Fig. 16. Effect of tuning on the transverse electric field.

mid-cell on the frequency shift is more significant than the first or the third cells, and the amount of the shift is larger at higher frequencies.

The cell misalignment disrupts the field flatness where this negative effect can be treated via mechanical tuning. Accordingly, Fig. 15 illustrates the compensation of the electric-field decrease at the center of the cavity using perturbation tuning for the first misaligned cell of the 9-cell cavity. On the other hand, the increase of the transverse electric field that occurs due to alignment error in the first cell does not disappear via mechanical tuning, but rather increases, see Fig. 16.

Mechanical tuning is a method based on the change in the size of the cell whose electrical field is lower than the field of other cells. In this way, the electric field of the respective cell can be adjusted. In our case, the effects of a $0.8-\mathrm{mm}$ alignment error in the first cell are eliminated by reducing the "xlen" parameter to approximately $99 \%$ of its initial value.

The improvement of the field flatness recovers values of the parameters which are dependent on the electric-field variations, for example, $R_{\mathrm{sh}} / Q, E_{\mathrm{peak}} / E_{\mathrm{acc}}$, and $B_{\text {peak }} / E_{\mathrm{acc}}$. On the 

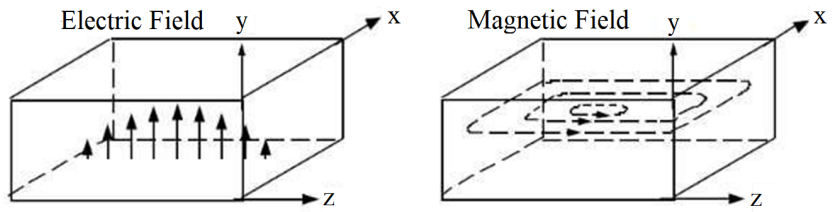

Fig. 17. Electric- and magnetic-field distribution of the $\mathrm{TE}_{101}$ mode.

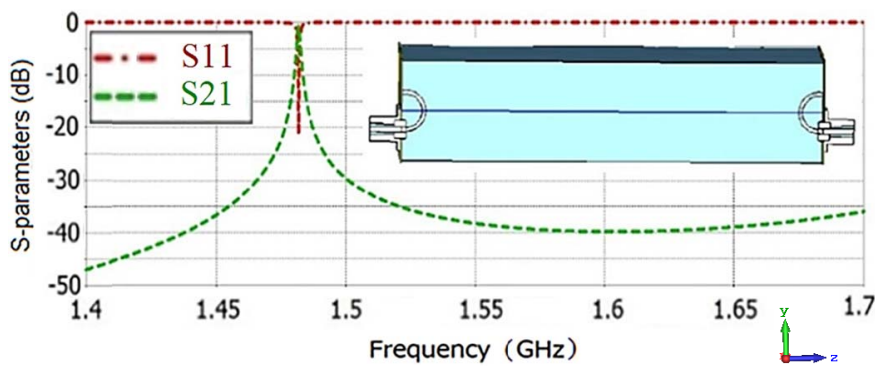

Fig. 18. S-parameters of the prototype rectangular cavity.

other hand, the geometry factor does not improve even after tuning because the surface to be integrated increases. It can be seen from Fig. 8 that the geometry factor is reduced from 99.71 to $99.625 \Omega$ due to 0.8 - $\mathrm{mm}$ misalignment. However, after perturbation tuning, this value falls below $99.3 \Omega$.

\section{SURFACE IMPEDANCE CONCEPT}

In principle, a conducting structure with a rough surface can be represented by a simpler structure having a complex-valued impedance function defined on its surface. The impedance is a function of space coordinates since the conductivity varies along the structure [1], [2]. In this section, we investigate the applicability of a surface impedance approach for the rough copper cavity walls. To do this, we use a simple rectangular cavity whose $\mathrm{TE}_{101}$ mode operates at $1.48 \mathrm{GHz}$ with the field distributions of the $\mathrm{TE}_{101}$ mode of a rectangular cavity shown in Fig. 17.

Note that, the $\mathrm{x}-,, \mathrm{y}-, \mathrm{z}$-axis in Fig. 17 are the same as in Fig. 18, while the electromagnetic wave is assumed to be propagating in the z-direction. The cavity is excited via n-type connectors attached to small loop antennas to form an input coupler structure for low-power experiments, see Fig. 18. Here, the blue line inside the cavity indicates a straight measurement line, which is located between the centers of the antennas, for the sampling of the electric-field distribution. Fig. 18 is an output of a frequency-domain solver that returns the S-parameters of the structure and shows that the cavity is appropriately excited via input couplers.

Now we are ready to investigate the modeling of surface roughness with the impedance approach. For this, 240 uniformly distributed defects with Gaussian shape having a maximum amplitude of $100 \mu \mathrm{m}$ are located on the designed rectangular cavity walls to represent a relatively large physical roughness. The total width of the defect is chosen to cover twothirds of each surface. One-third of the surface is adjusted to have inward defects, one-third has outward defects, and the remaining part is chosen as a smooth surface. We note that half of the defects are inward while the other half are outward, and the frequency shift is not the main concern in
TABLE IV

LOSS COMPARISON

\begin{tabular}{cc}
\hline \hline Parameter & Value $(\mathrm{mW})$ \\
\hline Input Power & 500 \\
Metal loss with smooth surface & 58.8 \\
Metal loss with physical roughness & 66.0 \\
Metal loss with roughness model & 65.9 \\
\hline \hline
\end{tabular}

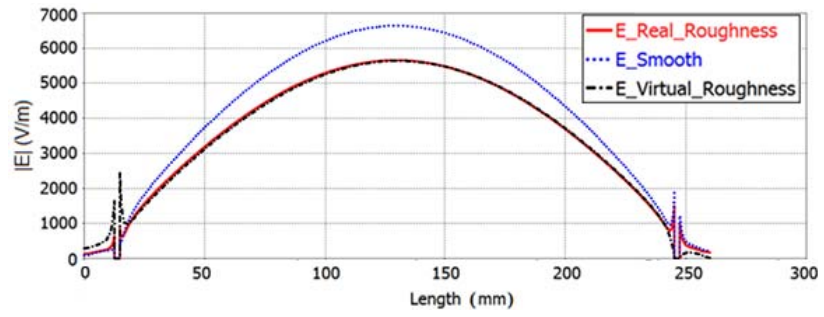

Fig. 19. Electric-field comparison for the $\mathrm{TE}_{101}$ mode.

this scenario. Afterward, the total power loss on the cavity walls and the electric-field magnitude on the measurement line are calculated. In the next empirical step, we search coefficients of the surface impedance defined on a perfectly smooth version of the cavity, which provides the equivalent total power loss and the electric field. Surface impedance is obtained with the help of the tabulated surface impedance tool of CST based on Huray's snowball model. Since this model has been proposed for microstrip lines, one should note that the formulations in [1] and [2] are not applicable for the cavities except for empirical methods. We show that the surface roughness of the cavities can also be represented via impedance functions in this article provided that the power loss and electric fields of the physically rough and modeled (via surface impedance) cavities are matched. In Table IV, the results of the total power loss calculated for the perfectly smooth, physically rough, and surface impedance modeled cavity walls are listed. Here, physical roughness refers to the cavity walls that have 240 defects, while the roughness model indicates tabulated surface impedance on the cavity walls.

It is evident from the table that proper empirical choices of the coefficients for the impedance model yield approximately the same total power loss value as in the case of a physically rough cavity. Moreover, the electric fields on the measurement line are compared in Fig. 19 such that the physical roughness and roughness model agree very well. The ripples at around 15 and $240 \mathrm{~mm}$ are due to the impedance contributions of the loop antennas. As a result, the peak value of the electric field in the cavity decreases if a roughness exits on the walls, and this would lead to a decrease in the accelerating gradient for the cavities used for particle acceleration.

Modeling of the physical roughness with surface impedance provides significant computational efficiency. For such simulations, finite-difference time domain or FEM-based on full Maxwell system solvers should mesh structures very accurately in order to take into account the micrometer level of roughness. Otherwise, the surface roughness effect would not be or be wrongly observed in the simulation output. 


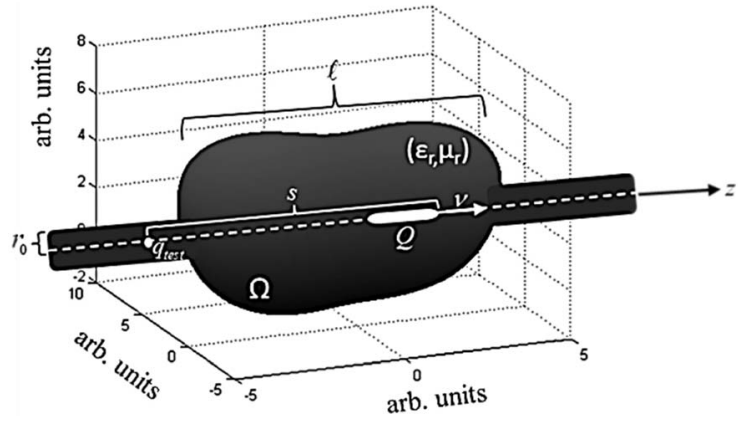

Fig. 20. Wake potential calculation geometry.

As a remedy for this important problem, we propose using surface impedance modeling for the accurate simulations of the rough cavities as a conclusion of this test study. In this way, the same field distribution inside the rectangular cavity is obtained in $\sim 20$ min even though the runtime reaches up to $\sim 12 \mathrm{~h}$ for the physically rough structure simulation. The value of this result becomes even more dramatic when a complex geometry, for example, a multi-cell elliptical cavity and its closely distributed resonant modes in a frequency spectrum, are considered. Along this line, Section V is devoted to showing the applicability of wakefields of the 9-cell prototype elliptical cavity with surface impedance.

\section{WAKe Potential AND IMPEDANCES}

As a bunch of charged particles passes inside an accelerator structure, electromagnetic fields can be generated due to a change in the cross section, surface resistivity variations of the beam pipe, roughness on the walls, or existence of an electron cloud formation inside the beam pipe. The wakefield solver of the CST-Microwave Studio (MWS) program is based on the finite integration technique rather than FEM.

Due to causality, those fields should exist behind the beam and, therefore, they are called wakefields. Wakefields can be trapped inside the structure or propagate through the beam pipe and can cause instability for the trailing particles so that accurate calculations are needed. In this context, wake potentials basically represent the effect, which is seen by following the test particle with the charge $q_{\text {test }}$, considering a sample scenario in Fig. 20. Here, the beam pipes are attached to the cavity structure given in Fig. 1. Inside the cavity, the Lorentz force acts

$$
\mathbf{F}(x, y, z, t)=q_{\text {test }}(\mathbf{E}(x, y, z, t)+\mathbf{v} \times \mathbf{B}(x, y, z, t))
$$

on a test particle due to the electromagnetic fields excited by the bunch, which has a total charge $Q$ passing through the center of beam pipe with the velocity of $\mathbf{v}=\mathrm{v}_{0} \mathbf{e}_{\mathbf{z}},\left(\mathrm{v}_{0}=\beta c\right)$, where $\mathbf{e}_{\mathbf{z}}$ is the unit vector in the positive z-direction. If we assume that the bunch is infinitely short and consider only the longitudinal force at the center of the beam pipe

$$
F_{||}(z, t)=q_{\text {test }} E_{z}(z, t)
$$

then the energy gain of the test particle can be calculated via

$$
U_{\|}(s)=\left.\int_{0}^{l} d z F_{\|}(z, t)\right|_{t=\frac{s+z}{v}}
$$

since the transverse force seen by the test particle affects the deflecting kicks, where $s$ is the distance between the bunch and the test particle. Then, the definition of the longitudinal wake function $w_{\|}(s)$ is given as

$$
w_{\|}(s)=-\frac{U_{\|}(s)}{q_{\text {test }} Q} .
$$

Accordingly, the Fourier transform of the wake function is called the wake impedance or coupling impedance. The convolution of the wake function with the line charge density is defined as the wake potential which is generally expressed in the literature by normalizing its value to the total bunch charge in V/C [5], [16]-[18]. Accordingly, the longitudinal wake potential $W_{\|}$is given

$$
W_{\|}(s)=\int_{0}^{\infty} d s^{\prime} w_{\|}\left(s^{\prime}\right) \rho_{l}\left(s-s^{\prime}\right)
$$

where $\rho_{l}$ represents the normalized line density, and the integral's lower limit is zero due to causality [6]. Even though the wake potential represents the instability in the time domain, the impedance is a frequency-dependent function. For more detailed information on wake potentials and impedances, we suggest [5], [6], and [19] to the reader. One can find a computational study related to the wakefield in [20], similar to this article.

As was illustrated in Section IV, one can use the tabulated surface impedance, which is a simulation tool provided by CST-MWS, to investigate the effects of the surface roughness on conductor loss or electromagnetic-field distributions and eventually the wakes. Along this line, the effects of surface roughness on wake potentials and impedances will be investigated. For this aim, a 10-mm long, Gaussian, ultrarelativistic pencil beam with the total charge $Q=10 \mathrm{nC}$ consisting of protons is considered to be traveling on the beam axis of the prototype 9-cell elliptical cavity in the wakefield solver. Furthermore, the surface impedance value $0.53+\mathrm{j} 0.53$ $\Omega$, which can be considered an exaggerated value, is chosen to represent an arbitrary roughness value. Note that the same process as in Table III is performed for wakefield solutions, and wake results converge to analytical solutions in the case of $\sim 5$ million hexahedral mesh cells in the computational domain.

Simulation results for the impedance calculations using perfectly smooth and surface impedance modeled cavities are illustrated in Figs. 21 and 22, respectively. Here, the frequency spectrum is chosen to show the impedances of the $\pi$-mode (the first harmonic) and the next higher mode (the second harmonic) such that the maximum peak is observed at the $\pi$-mode at $3.9008 \mathrm{GHz}$. The wake impedance for the smooth surface is found as $270.35 \mathrm{k} \Omega$, while for the surface having roughness, the value decreases to $170.66 \mathrm{k} \Omega$. Similarly, the impedance in the second harmonic is decreased to $80.31 \mathrm{k} \Omega$ while it is equal to $163.71 \mathrm{k} \Omega$ for the smooth cavity walls.

Wake potentials, given in Figs. 23 and 24, are calculated in the cavity by considering the same scenarios, for example, the same surface impedance $0.53+\mathrm{j} 0.53 \Omega$ is used on the cavity walls, for Fig. 24. In Fig. 23, oscillations in the wake potential have a larger magnitude compared to simulation results for 


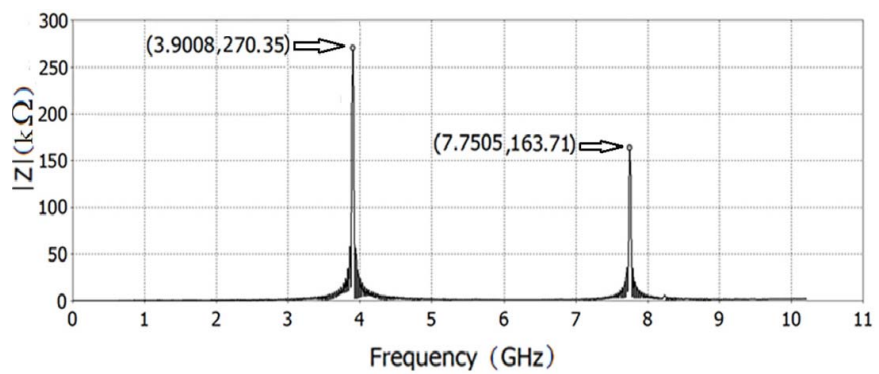

Fig. 21. Wake impedance with a smooth surface.

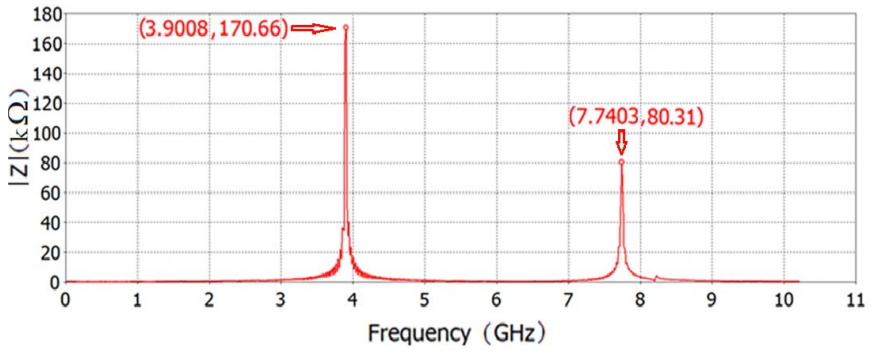

Fig. 22. Wake impedance with a rough surface.

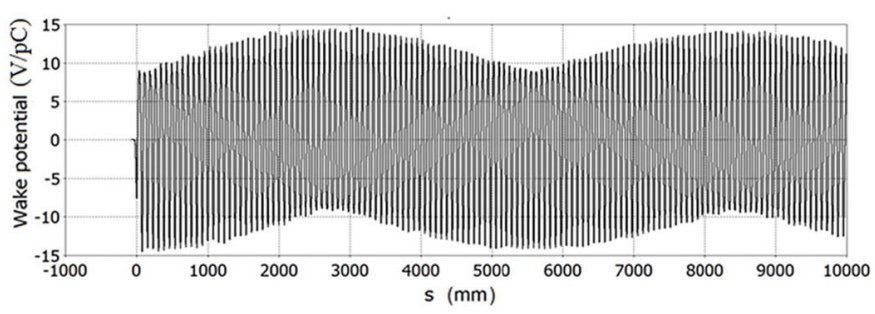

Fig. 23. Longitudinal wake potential with a smooth surface.

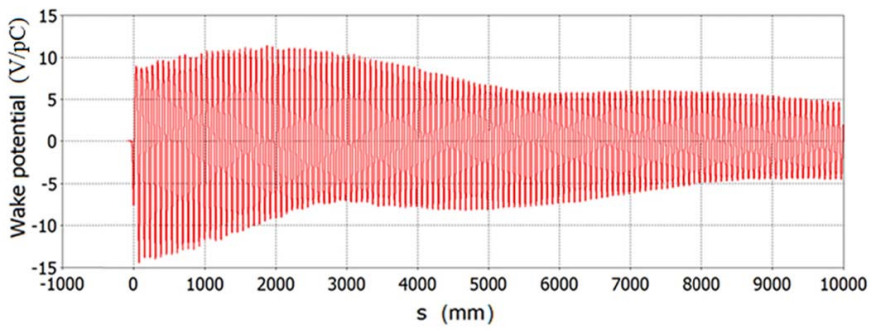

Fig. 24. Longitudinal wake potential with a rough surface.

the rough cavity in Fig. 24, where the wake is attenuating faster due to the power loss on the cavity wall. Since the wake potential is generally an unwanted effect for the particles, one can conclude that wake potential can be decreased more quickly using the rough surface with the cost of decreasing the accelerating gradient. All simulations are carried out under the assumption that the cavity walls are not superconductors as presented in [21].

\section{EXPERIMENTAL RESULTS}

In the experiments, a 3-cell elliptical cavity, whose $\pi$-mode oscillates at $3.9 \mathrm{GHz}$, is considered for the sake of production simplicity instead of the 9-cell cavity presented in Section III.
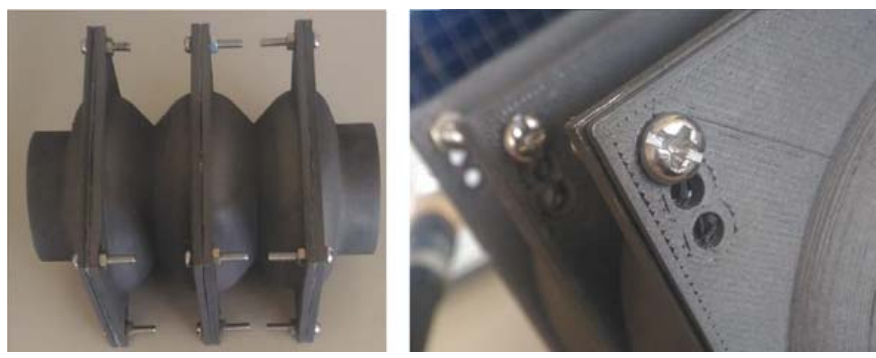

Fig. 25. 3-D printed 3-cell cavity and screw holes.

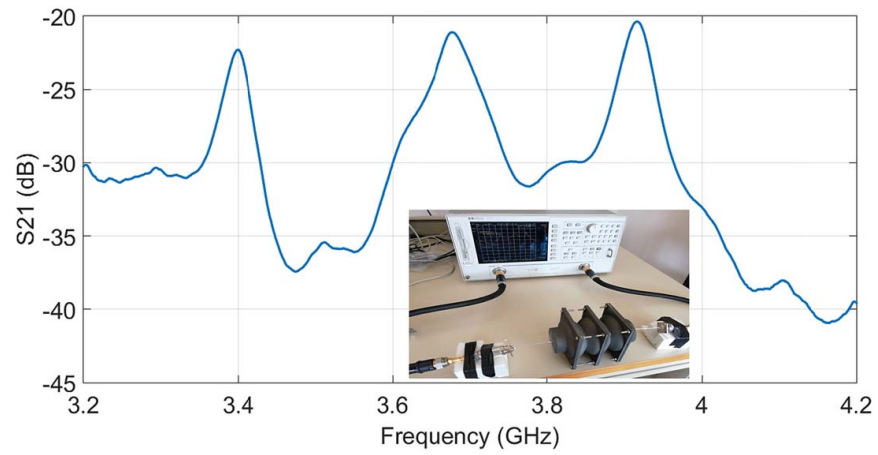

Fig. 26. Transmission measurement.

Furthermore, a larger iris radius is chosen for the 3-cell cavity to increase the cell-to-cell coupling and to cure a possible mode interference problem during measurements with a cavity having a low-quality factor. On the other hand, an increase in cell-to-cell coupling makes it difficult to observe the effects due to geometric variations [22], [23]. Accordingly, the misalignment value is increased to an exaggerated value, for example, 4.5 and $9 \mathrm{~mm}$. The image of the cavity after being fabricated and coated with nickel can be seen in Fig. 25.

In the 3-cell cavity, the equator diameter is chosen as 7 $\mathrm{cm}$, and the diameter of the iris is approximately $5 \mathrm{~cm}$, such that the cell-to-cell coupling is increased by $14.1 \%$. The prototype cavity is fabricated with a 3-D printer and became conductive with the help of conductive nickel spray and aluminum tape. Since the frequency shift and geometry factor are not dependent on the conductivity of the structure, we could use this structure for our experiments. Also, electroplating has been applied to the nickel-coated cavity, and individual measurements have been taken. For a 3-cell cavity, we need to produce two half-end-cells (including beam-pipes) and two mid-dumb-bells. This enables experiments with the equator alignment. There are three screw holes on each structure, and the distance between the centers of each screw hole is $4.5 \mathrm{~mm}$.

The transmission and reflection measurements are performed via probe and loop antennas by using nickel-coated and copper electro-cell elliptical cavities, respectively. Three resonances are observed during $S_{21}$ and $S_{11}$ measurements, which can be seen in Figs. 26 and 27. The first peak in Fig. 26 refers to the zero mode, and the third one refers to the $\pi$-mode. The second one represents the mode, whose electricfield component has two peaks along the acceleration axis. To reduce the external quality factor up to approximately zero, 


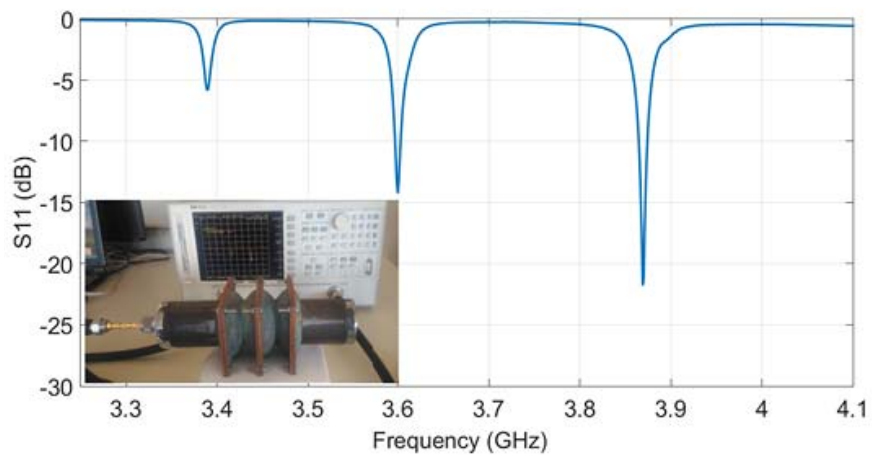

Fig. 27. Reflection measurement.

TABLE V

GeOMETRY FACTOR MEASUREMENT

\begin{tabular}{cccccc}
\hline \hline $\begin{array}{c}\text { Meas. } \\
\text { Type }\end{array}$ & Aligned & $\begin{array}{c}4.5 \mathrm{~mm} \\
\text { on cell } \\
\# 1\end{array}$ & $\begin{array}{c}9 \mathrm{~mm} \\
\text { on cell } \\
\# 1\end{array}$ & $\begin{array}{c}4.5 \mathrm{~mm} \\
\text { on cell } \\
\# 2\end{array}$ & $\begin{array}{c}9 \mathrm{~mm} \\
\text { on cell } \\
\# 2\end{array}$ \\
\hline Transmission & $315 \Omega$ & $278 \Omega$ & $199 \Omega$ & $261 \Omega$ & $187 \Omega$ \\
Transmission & $303 \Omega$ & $270 \Omega$ & $205 \Omega$ & $249 \Omega$ & $181 \Omega$ \\
Reflection & $315 \Omega$ & $282 \Omega$ & $216 \Omega$ & $252 \Omega$ & $192 \Omega$ \\
\hline \hline
\end{tabular}

one has to ensure that the probes, which are located symmetrically, should couple the electromagnetic fields weakly to the cavity [24]. In Fig. 27, the $S_{11}$ result of the critical coupling measurement is given for the correctly aligned cells. We note that due to the additional beam pipes and critically coupled antenna, the resonant frequencies are slightly shifted to lower values.

The experiment, seen in Fig. 26, is repeated as two sets for different misalignment values for cell \#1 and cell \#2. The conductivity of the cavity walls is reconstructed via simulation as $1700 \mathrm{~S} / \mathrm{m}$ at $3.9 \mathrm{GHz}$. Afterward, the geometry factor can be obtained from the measured $Q$ value and surface resistance. Similarly, the conductivity of the cavity walls is found as $10^{4}$ $\mathrm{S} / \mathrm{m}$ for the electroplated cavity, which is used in the reflection measurement. Since the geometry factor does not depend on the material of the cavity, we observe the consistency in the experimental results. One can see from Table V that when the misalignment value increases, the geometry factor decreases, and the mid cell is seen to be more effective than the end cell on the geometry factor. The differences in the measured geometry factor with two different methods are related to the accuracy in our experiments. However, the mismatch is sufficiently small such that we can conclude that the geometry factor has similar variations in both cases. We note that the geometry factor is the product of quality factor and surface resistance having the unit $\Omega$.

The same procedure is applied to determine the frequency shift. At this point, we remind the reader that the frequency shifts stay in the kilohertz range as presented in Fig. 14. Although the amount of decrease on the geometry factor for a very high misalignment value is significant, the frequency shift is still insignificant. The $S_{21}$ variation of the $\pi$-mode for the different misalignment values is shown in Fig. 28. The

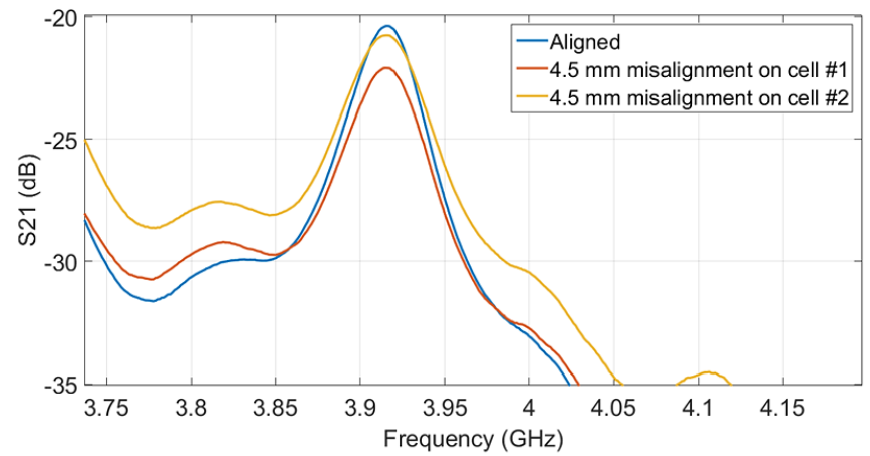

Fig. 28. Zoomed version of $\pi$-mode peaks.

change in frequency is very small to be seen for the large misalignment values considered in our experiments.

\section{CONCLUSION}

The fabrication process of the accelerating cavities can be challenging, depending on the desired operating parameters. Misalignment during assembling of the half-cells and precise etching of the cavity structure from the bulk material play significant roles such that both cases are studied in this article.

In the first part, it is shown using a 9-cell $(\pi$-mode: $3.9 \mathrm{GHz})$, a 3-cell $(\pi$-mode: $3.9 \mathrm{GHz})$, and another 3-cell ( $\pi$-mode: $2 \mathrm{GHz}$ ) elliptical cavities that cavity design parameters deteriorate when the amount of misalignment on any cell is increased. In the simulations, we observed that misalignment on the end-cells has a larger impact on the $R_{\mathrm{sh}} / Q, E_{\text {peak }} / E_{\text {acc }}$, and $B_{\text {peak }} / E_{\text {acc }}$ even though the misalignment on the mid-cell affects the geometry factor at most. The simulation result for the geometry factor is verified with the experiments. The behaviors of the $E_{\text {peak }} / E_{\text {acc }}$ and $B_{\text {peak }} / E_{\text {acc }}$ are expected since the field flatness of the cavity diverges from the optimal value. Also, we observed that transverse kick and geometry factor could not be corrected with mechanical tuning, but the change in $R_{\mathrm{sh}} / Q, E_{\text {peak }} / E_{\text {acc }}$, and $B_{\text {peak }} / E_{\text {acc }}$ values can be compensated.

Second, we show that a surface roughness on the cavity walls can be represented as a complex-valued surface impedance. For this, a rectangular and ideally smooth copper test cavity is excited via loop antennas to have a realistic power transfer model, unlike in the homogeneous eigenvalue problem solver calculations. Then, the acquirability of the approximately equal field distributions and power loss values using both a smooth cavity having surface impedance on its walls and a cavity whose walls are physically rough are illustrated. A non-smooth cavity, which is modeled with surface impedance, is employed for the wakefield calculations. It is found that wake potentials for the rough cavities attenuate faster due to power loss on the cavity while the accelerating gradient decreases.

Finally, it is thought that the results presented in this article will give hints for modeling and understanding mechanical imperfections and its effects on the particle accelerations. 


\section{ACKNOWLEDGMENT}

The authors would like to thank Dr. Ing. F. Caspers at CERN for his valuable advice, and to the editor and the anonymous reviewers of the journal for their constructive remarks and helpful suggestions which improve the quality of this article.

\section{REFERENCES}

[1] G. Gold and K. Helmreich, "Surface impedance concept for modeling conductor roughness," in Proc. IEEE MTT-S Int. Microw. Symp. Dig., Phoenix, AZ, USA, May 2015, pp. 1-4.

[2] K. Lomakin, G. Gold, and K. Helmreich, "Transmission line model for rectangular waveguides accurately incorporating loss effects," in Proc. IEEE 21st Workshop Signal Power Integrity, Baveno, Italy, May 2017, pp. $1-4$.

[3] P. G. Huray, O. Oluwafemi, J. Loyer, E. Bogatin, and X. Ye, "Impact of copper surface texture on loss: A model that works," in Proc. DesignCon, 2010, pp. 462-483.

[4] L. Simonovich, "Practical method for modeling conductor roughness using cubic close-packing of equal spheres," in Proc. IEEE Int. Symp. Electromagn. Compat., Ottawa, ON, Canada, Jul. 2016, pp. 917-920.

[5] V. Vaccaro et al., "Wake fields and impedance," in Proc. CAS Adv. School Accel. Phys., Rhodes, Greece, 1994, pp. 1-128.

[6] B. Zotter and S. A. Kheifets, Impedances and Wakes in High-energy Particle Accelerators. Singapore, World Scientific Publishing, 1998.

[7] P. Schmüser, "Basic principles of RF superconductivity and superconducting cavities," in Proc. CERN Accel. School Lecture Notes, 2003, pp. 1-19.

[8] CST AG-Computer Simulation Technology, Darmstadt, Germany. CST MICROWAVE STUDIO. (2018). [Online]. Available: http://www.cst.com

[9] A. Karatay and F. Yaman, "Electromagnetic simulations of mechanical imperfection for elliptical cavities," presented at the 34th Int. Phys. Congr. Turkish Phys. Soc., Bodrum, Turkey, Sep. 2018.

[10] L. Xiao et al., "Modeling imperfection effects on dipole modes in TESLA cavity," in Proc. IEEE Part. Accel. Conf., Albuquerque, NM, USA, Jun. 2007, pp. 2454-2456.

[11] L. Lee et al., "Shape determination for deformed cavities," SLAC, Menlo Park, CA, USA, Tech. Rep. PUB-12141, 2007.
[12] T. Czarski, K. T. Pozniak, R. S. Romaniuk, and S. Simrock, "TESLA cavity modeling and digital implementation with FPGA technology solution for control system development," Proc. SPIE, vol. 5484, Jul. 2004, Art. no. 568854.

[13] B. Aune et al., "Superconducting TESLA cavities," Phys. Rev. Special Topics-Accel. Beams, vol. 3, Sep. 2000, Art. no. 092001.

[14] W. Ebeling et al., "Superconducting RF-cavities for a 30-GeV PETRA storage ring," IEEE Trans. Nucl. Sci., vol. NS-30, no. 4, pp. 3357-3359, Aug. 1983.

[15] W. Singer, "Fabrication of elliptical SRF cavities," Superconductor Sci. Technol., vol. 30, no. 3, Jan. 2017, Art. no. 033001.

[16] K. Fujita, H. Kawaguchi, I. Zagorodnov, and T. Weiland, "Time domain wake field computation with boundary element method," IEEE Trans. Nucl. Sci., vol. 53, no. 2, pp. 431-439, Apr. 2006.

[17] A. Novokhatski, "A new Green's function for the wake potential calculation of the SLAC S-band constant gradient accelerating section," Nucl. Instrum. Methods Phys. Res. A, Accel., Spectrometers, Detectors Associated Equip., vol. 684, pp. 46-51, Aug. 2012.

[18] A. Tsakanian, M. Dohlus, and I. Zagorodnov, "Short range wake potentials of FLASH resistive tapered collimator and European XFEL undulator intersection," Nucl. Instrum. Methods Phys. Res. A, Accel., Spectrometers, Detectors Associated Equip., vol. 659, pp. 9-13, Dec. 2011.

[19] T. Weiland and R. Wanzenberg, "Wake fields and impedances," in Proc. CCAS-CAT-CERN Accelerator School, vol. 1. Indore, India: Advanced Accelerator Physics and Technology Course, 1993.

[20] K. Fujita, H. Kawaguchi, T. Weiland, and S. Tomioka, "Threedimensional wake field computations based on scattered-field time domain boundary element method," IEEE Trans. Nucl. Sci., vol. 56, no. 4, pp. 2341-2350, Aug. 2009.

[21] B. Xiao et al., "RF design of normal conducting $704 \mathrm{MHz}$ and 2.1 GHz cavities for LEReC Linac," in Proc. IPAC, Busan, Korea, 2016, pp. 1-6.

[22] A. Sulimov, "RF analysis of equator welding stability for the European XFEL cavities," in Proc. SRF, Whistler, BC, Canada, 2015, pp. 1272-1273.

[23] A. Sulimov et al., "RF analysis of electropolishing for EXFEL cavities production at ettore Zanon SpA," in Proc. 28th Linear Accel. Conf., East Lansing, MI, USA, vol. 35, Sep. 2016, pp. 544-546.

[24] D. M. Pozar, Microwave Engineering. New York, NY, USA: Wiley, 1990. 